\title{
THE NUMERICAL FUNCTION OF A *-REGULARLY VARYING SEQUENCE
}

\author{
Dragan Đurčić and Aleksandar Torgašev*
}

\begin{abstract}
In this paper, we impose some conditions under which there is a close relation between the asymptotic behaviour of a $*$-regularly varying sequence and the asymptotic behaviour of its numerical function $\delta_{c}(x), x>0$.
\end{abstract}

\section{Introduction and results} we have

$$
\bar{k}_{c}(\lambda)=\varlimsup_{n \rightarrow+\infty} \frac{c_{[\lambda n]}}{c_{n}}<+\infty, \quad \lambda>0 .
$$

The class of all $O$-regularly varying sequences is denoted $O R V$.

An $O$-regularly varying sequence $\left(c_{n}\right)$ is called $*$-regularly varying [6], if it is nondecreasing, and if

$$
\lim _{\lambda \rightarrow 1+} \bar{k}_{c}(\lambda)=1
$$

The class of all $*$-regularly varying sequences is denoted $* \mathrm{RV}$.

The above two classes of sequences represent the important objects in the sequential theory of regular variability in the Karamata sense [1], and in particular in the theory of statements of Tauberian type [4], as well as in some other parts of qualitative analysis of divergent processes [7].

The class $K_{c}^{*}[5]$, consists of all *-regularly varying sequences which satisfy the condition

$$
\underline{k}_{c}(\lambda)=\underline{\lim }_{n \rightarrow+\infty} \frac{c_{[\lambda n]}}{c_{n}}>1, \quad \lambda>1 .
$$

AMS (MOS) Subject Classification 1991. Primary: 26A12.

Key words and phrases: Regularly varying sequence, numerical function.

${ }^{*}$ Research supported by Science Fund of Serbia under Grant 1457. 
Notice that, in particular, the class $K_{c}^{*}$ contains all nondecreasing regularly varying sequences in the Karamata sense [1] whose index $\rho>0$, and also all sequences whose general term is the $n$-th $(n \in N)$ partial sum of a $*$-regularly varying sequence, but does not contain slowly varying sequences in the Karamata sense [1].

If next, $\left(c_{n}\right)$ is an increasing sequence of positive numbers, then its numerical function $\delta_{c}(x), x>0$, is defined by $\delta_{c}(x)=\sum_{c_{n} \leq x} 1, x>0$.

We shall prove several statements about the mentioned classes.

By $\asymp$ we shall denote the weak, while by $\sim$ the strong asymptotic equivalence.

Theorem 1. Let $\left(c_{n}\right)$ be an increasing sequence from the class $K_{c}^{*}$ and assume that $g:[1,+\infty) \mapsto(0,+\infty)$ is a continuous and increasing function. Then we have

$$
c_{n} \sim g(n), \quad n \rightarrow \infty
$$

if and only if

$$
\delta_{c}(x) \sim g^{-1}(x), \quad x \rightarrow+\infty .
$$

Notice that if $\left(c_{n}\right)$ is an arbitrary increasing sequence of positive number which is not in the class $K_{c}^{*}$, it is easy to construct a contionuous and increasing function $g:[1,+\infty) \mapsto(0,+\infty)$, so that (4) is true but not (5) or, (5) is true but not (4).

Corollary 1. Let $\left(c_{n}\right)$ be an increasing sequence from the class $K_{c}^{*}$, and $\left(d_{n}\right)$ be an increasing sequence of positive numbers. Then we have

$$
c_{n} \sim d_{n}, \quad n \rightarrow \infty
$$

if and only if

$$
\delta_{c}(x) \sim \delta_{d}(x), \quad x \rightarrow \infty .
$$

Corollary 1 follows easily from the theorem above.

Corollary 2. Let $\left(c_{n}\right)$ be an increasing sequence from the class $K_{c}^{*}$ and let $g:[1,+\infty) \mapsto(0,+\infty)$ be a continuous and increasing function. If (4) holds, then we have

$$
\sum_{c_{n} \leq x} c_{n} \asymp x g^{-1}(x), \quad x \rightarrow+\infty .
$$

Corollary 3. Let $\left(c_{n}\right)$ be an increasing sequence from the class $K_{c}^{*}$ and $\left(d_{n}\right)$ be an increasing sequence of positive numbers. If (4') holds, then we have

$$
\sum_{c_{n} \leq x} c_{n} \asymp \sum_{d_{n} \leq x} d_{n}, \quad x \rightarrow+\infty .
$$




\section{Proofs of statements}

Proof of the theorem. Consider the function $f(x), x \geq 1$, for which we have $c_{n}=f(n)$. It is obviously linear on intervals $[n, n+1], n \in N$.

For any $\delta>0$, there is some $n_{0}=n_{0}(\delta) \in N$, so that for all $n \geq n_{0}$ we have $1 \leq 1+\frac{1}{n} \leq \delta+1$, so that we find $1 \leq \varlimsup_{n \rightarrow+\infty} \frac{c_{n+1}}{c_{n}} \leq \bar{k}_{c}(1+\delta)$. Since by assumption $\left(c_{n}\right) \in K_{c}^{*}$, it is $*-$ regularly varying, so that $\lim _{n \rightarrow \infty} \frac{c_{n+1}}{c_{n}}=1$. If (4) holds true, then we have $f(x) \sim g(x), x \rightarrow+\infty$, because for all $n \leq x<n+1$, $n \in N$, we have that

$$
\frac{c_{n}}{c_{n+1}} \cdot \frac{c_{n+1}}{g(n+1)} \leq \frac{f(x)}{g(x)} \leq \frac{c_{n}}{g(n)} \cdot \frac{c_{n+1}}{c_{n}} .
$$

Next, let for any $\lambda>0, \bar{k}_{f}(\lambda)=\varlimsup_{x \rightarrow+\infty} \frac{f(\lambda x)}{f(x)}$. Then for every $\delta>0$ we have

$$
\begin{aligned}
\bar{k}_{c}(\lambda) & \leq \bar{k}_{f}(\lambda) \leq \varlimsup_{x \rightarrow+\infty} \frac{f([\lambda x]+1)}{f([x])} \leq \\
& \leq \overline{\lim }_{x \rightarrow+\infty} \frac{c_{[\lambda[x]]}}{c_{[x]}} \cdot \overline{\lim }_{x \rightarrow+\infty} \frac{c_{[\lambda x]+1}}{c_{[\lambda[x]]}} \leq \\
& \leq \bar{k}_{c}(\lambda) \cdot \bar{k}_{c}(1+\delta),
\end{aligned}
$$

because

$$
\lim _{x \rightarrow+\infty} \frac{[\lambda x]+1}{[\lambda[x]]}=1+.
$$

This means that for every $\lambda>0$ we have $\bar{k}_{c}(\lambda)=\bar{k}_{f}(\lambda)$.

If we next redefine $f(x)$ by $f(0)=0$, and on the interval $[0,1]$ as a linear function, then we have that $f \in K_{c}^{*}$ (see [5]). If we in a similar way redefine $g(x)$ for $0 \leq x<1$, and we suppose (4), then by [3] we have

$$
f^{-1}(x) \sim g^{-1}(x), \quad x \rightarrow+\infty .
$$

Since $\delta_{c}(x)=\left[f^{-1}(x)\right], x>0$, we obtain (5).

Conversely, supposing that (5) holds true, then with the so redefined functions $f$ and $g$ we have (7). Since $f \in K_{c}^{*}$, we get $f(x) \sim g(x), x \rightarrow+\infty$, so that we obtain (4).

Remark. If $\left(c_{n}\right)$ is an increasing and unbounded $*$-regularly varying sequence, out the class $K_{c}^{*}$, then (5) implies (4) for every function $g$ described in the Theorem. But it is not difficult to see that there is a function $g$ which has properties from the Theorem, such that (4) does not implies (5).

If a sequence $\left(c_{n}\right)$ is increasing and unbounded, and it is not $*$-regularly varying, it is not clear if, in the general case, (4) and (5) are equivalent to each other for an arbitrary function $g$ described in the Theorem. 
Proof of Corollary 2. By assumptions, we have that

$$
\sum_{c_{n} \leq x} c_{n}=\int_{0}^{x} t d \delta_{c}(t) \leq x \delta_{c}(x), \quad x>0 .
$$

On the other side, we have

$$
\sum_{c_{n} \leq x} c_{n} \geq \int_{x / 2}^{x} t d \delta_{c}(t) \geq \frac{x}{2}\left(\delta_{c}(x)-\delta_{c}\left(\frac{x}{2}\right)\right), \quad x>0 .
$$

Since $\left(c_{n}\right) \in K_{c}^{*}$ we have that $\underline{k}_{c}(\lambda)>1, \lambda>1$, so that $\underline{k}_{\delta_{c}}(2)>1$. In other words, $\bar{k}_{\delta_{c}}\left(\frac{1}{2}\right)<1$. Next, define $p=1-\bar{k}_{\delta_{c}}\left(\frac{1}{2}\right)$. Then for all $x \geq x_{0}$ we have that

$$
\frac{p}{4} \leq \frac{\sum_{c_{n} \leq x} c_{n}}{x \delta_{c}(x)} \leq 1
$$

so that $\sum_{c_{n} \leq x} c_{n} \asymp x \delta_{c}(x), x \rightarrow+\infty$. By assumptions of the colollary, and the Theorem, we have that then $\delta_{c}(x) \sim g^{-1}(x), x \rightarrow+\infty$, so that (6) holds true.

Finaly, Corollary 3 is a direct consequence of the Theorem and the Corollary 2.

\section{References}

[1] N. H. Bingham, C. M. Goldie, J. L. Teugels, Regular Variation, Cambridge Univ. Press, Cambridge, 1987.

[2] D. Djurčić, V. Božin, A proof of a S. Aljančić hypothesis on O-regularly varying sequences, Publ. Inst. Math. (Beograd) 62 (76) (1997), 46-52.

[3] D. Djurčić, A. Torgašev, Strong Asymptotic Eqivalence and Inversion of Functions in the class $K_{c}$, Journal Math. Anal. Appl. 255 (2001), 383-390.

[4] W. Kratz, U. Stadtmüller, Tauberian Theorems for $J_{p}$-Summability, Journal Math. Anal. Appl. 139 (1989),362-371.

[5] M. Matuszewska, On a generalization of regularly increasing functions, Studia Math. 24 (1964), 271-279.

[6] Č. V. Stanojević, O-regularly varying convergence moduli of Fourier and Fourier-Stieltjes series, Math. Ann. 279 (1987), 103-115.

[7] H. Tietz, R. Trautner, Tauber-Sätze für Potenzreihenverfahren, Arch. Math. 50 (1988), 164-174.

First author: Technical Faculty, Svetog Save 65, 32000 Čačak, Serbia \& Montenegro
Second author: Mathematical Faculty, Studentski trg 16a, 11000 Belgrade, Serbia \& Montenegro. Received: January 15, 2002. 